\title{
Diabetic Ketoacidosis: Clinical Characteristics and Precipitating Factors
}

\author{
Wajeeha Shahid $^{1}$, Faria Khan ${ }^{2}$, Aamir Makda ${ }^{1}$, Vinesh Kumar ${ }^{3}$, Sidra Memon ${ }^{1}$, Amber Rizwan ${ }^{4}$ \\ 1. Internal Medicine, Jinnah Sindh Medical University, Karachi, PAK 2. Internal Medicine, Dow University of Health \\ Sciences, Karachi, PAK 3. Internal Medicine, Ghulam Muhammad Mahar Medical College, Sukkur, PAK 4. Family \\ Medicine, Jinnah Postgraduate Medical Center, Karachi, PAK
}

Corresponding author: Amber Rizwan, amber_aljazeera109@hotmail.com

\begin{abstract}
Introduction: Diabetic ketoacidosis (DKA) is a complication of hyperglycemia. Through this study we plan to study the clinical features of DKA and precipitating factors responsible for DKA in type 1 and type 2
\end{abstract} diabetes.

Methods: This cross-sectional observational study was conducted in the emergency department of a tertiary care hospital in Sukkur, Pakistan from August 2019 to February 2020. Symptoms and precipitating factors were noted in a self-structured questionnaire.

Results: Out of 71 patients, 19 (26.7\%) patients had type 1 diabetes mellitus and 52 (73.3\%) patients had type 2 diabetes mellitus. The most common clinical symptom was nausea and vomiting (57.7\%), followed by pain in abdomen (42.2\%) and dehydration (42.2\%). We found that the most common precipitating factors were infections (69.0\%) and non-compliance to treatment (53.5\%). Among various infections, people commonly presented with pneumonia (38.7\%) and urinary tract infection (30.6\%).

Conclusion: Diabetic ketoacidosis presents with vague symptoms such as nausea, vomiting, and pain in abdomen. Characteristic findings of DKA such as Kussmaul breathing was present in limited patients. Infections in diabetic patients should be carefully monitored as they are the most common precipitating factors for DKA.

Review began 09/12/2020 Review ended 09/30/2020 Published 10/04/2020

() Copyright 2020 Shahid et al. This is an open access article distributed under the terms of the Creative Commons Attribution License CC-BY 4.0, which permits unrestricted use, distribution, and reproduction in any medium, provided the original author and source are credited.
Categories: Endocrinology/Diabetes/Metabolism, Internal Medicine

Keywords: diabetic ketoacidosis (dka), pakistan

\section{Introduction}

Diabetes mellitus (DM) is an endocrine disorder that leads to abnormal metabolism of blood glucose. It is a chronic disease that results in both short-term and long-term complications. Diabetes can lead to a number of complications such as hyperosmolar hyperglycemic state (HHS) and diabetic ketoacidosis (DKA) [1]. Due to hyperglycemic emergencies, the incidence of mortality ranges from $4 \%$ to $40 \%$ in developing countries [2].

DKA presents with vague symptoms such as nausea, vomiting, and abdominal pain. Other symptoms include increased thirst and urination. Kussmaul breathing (labored deep breathing) and fruity odor are specific signs present on examination of a patient with diabetic ketoacidosis [3]. Various precipitating factors of DKA are reported in studies, especially missed insulin dose and an ongoing infection [4]. Other precipitating factors include stressful events such as stroke, myocardial infarction, and trauma, as well as substance abuse [5]. The clinical outcome of DKA depends upon the patient's response to initial medical intervention, the precipitating factor for DKA, and biochemical values. Factors such as advanced age, bedridden state, and the use of mechanical ventilator are independent predictors associated with 30-day mortality [4].

Diabetes and its complications are both very prevalent in Pakistan. However, there is minimal literature on symptoms, precipitating factors, and outcome of patients with DKA. This study aims to assess the trend of clinical features of DKA and its precipitating factors in diabetic patients. This study may assist clinicians in early recognition of DKA, leading to its timely management.

\section{Materials And Methods}

This was a cross-sectional, observational study, conducted in the emergency department of a tertiary care hospital in rural Sindh, Pakistan. The study duration was August 2019 - February 2020 during which 71 participants were included in the study. Diagnostic criteria mentioned in the International Society for Paediatric and Adolescent Diabetes (ISPAD) Clinical Practice Consensus Guidelines 2018 were used as a reference for diagnosis and management of DKA [5]. According to guidelines, we diagnosed DKA through a triad of symptomatology and lab findings, i.e. hyperglycemia, ketosis, and acidemia (Figure 1). 


\section{Cureus}

\section{Diagnosis of Diabetic Ketoacidosis:}

1. Ketonaemia $>3.0 \mathrm{mmol} / \mathrm{L}$ or significant ketonuria (more than $2+$ on standard urine sticks)

2. Blood glucose $>11.0 \mathrm{mmol} / \mathrm{L}$ or known diabetes mellitus

3. Bicarbonate $\left(\mathrm{HCO3}^{-}\right)<15.0 \mathrm{mmol} / \mathrm{L}$ and/or venous $\mathrm{pH}<7.3$

\section{FIGURE 1: Diagnostic Criteria for Diabetic Ketoacidosis}

Clinical presentation and precipitating risk factors were noted in a self-administrated questionnaire. Stress to body was considered as any recent cardiovascular event such as myocardial infarction or cerebrovascular event such as stroke. A blood sample was taken at the time of admission and sent to the laboratory for ketone levels and bicarbonates. Blood glucose level was checked using a glucometer. Data were processed and analyzed using IBM SPSS Statistics for Windows, version 22.0 (IBM Corp., Armonk, NY, USA). Mean and standard deviation (SD) were calculated for continuous variables. Frequency and percentages were calculated for categorical variables.

\section{Results}

Out of 71 patients, 19 (26.7\%) patients had type 1 diabetes mellitus and 52 (73.3\%) had type 2 diabetes mellitus. Mean age of patients in this study was $52 \pm 11$ years. Mean age of participants for type 1 diabetes was $39 \pm 6$ years and for type 2 diabetes it was $58 \pm 11$ years. The mean duration of diabetes was $6 \pm 2$ years. There were 34 (47.8\%) males and 37 (52.2\%) females. The most common clinical symptoms were nausea and vomiting (57.7\%), followed by pain in abdomen (42.2\%) and dehydration (42.2\%). Kussmaul breathing was present in 10 (14.0\%) participants (Table 1).

\begin{tabular}{|c|c|c|}
\hline Symptoms & Number of patients $(n=71)$ & Percentage \\
\hline Nausea/vomiting & 41 & $57.7 \%$ \\
\hline Pain abdomen & 30 & $42.2 \%$ \\
\hline Dehydration & 30 & $42.2 \%$ \\
\hline Polyuria/polydipsia & 20 & $28.1 \%$ \\
\hline Altered sensorium & 18 & $25.3 \%$ \\
\hline Weakness & 12 & $16.9 \%$ \\
\hline Hypotension & 10 & $14.0 \%$ \\
\hline Kussmaul breathing & 10 & $14.0 \%$ \\
\hline
\end{tabular}

\section{TABLE 1: Symptoms of Diabetic Ketoacidosis}

The most common precipitating factors in this study were infections (69.0\%) and non-compliance to treatment (53.5\%) (Table 2). 


\section{Cureus}

\begin{tabular}{|c|c|c|}
\hline Precipitating factors & Number of patients $(n=71)$ & Percentage \\
\hline Infection & 49 & $69.0 \%$ \\
\hline Non-compliance to treatment & 38 & $53.5 \%$ \\
\hline Stress to Body & 10 & $10.0 \%$ \\
\hline First presentation & 7 & $9.8 \%$ \\
\hline Unknown & 7 & $9.8 \%$ \\
\hline
\end{tabular}

TABLE 2: Precipitating Factors Identified in Patients with Diabetic Ketoacidosis

Among various infections, pneumonia (38.7\%) and urinary tract infections (30.6\%) were most commonly reported (Table 3).

\begin{tabular}{|c|c|c|}
\hline Infections & Number of patients $(n=49)$ & Percentage \\
\hline Pneumonia & 19 & $38.7 \%$ \\
\hline Urinary tract infection & 15 & $30.6 \%$ \\
\hline Pulmonary tuberculosis & 5 & $10.2 \%$ \\
\hline Diabetic foot & 5 & $10.2 \%$ \\
\hline Gastrointestinal tract infection & 4 & $8.2 \%$ \\
\hline
\end{tabular}

TABLE 3: Infections Identified in Patients with Diabetic Ketoacidosis

\section{Discussion}

Diabetic ketoacidosis is associated with reduced level of functional insulin in the body. This reduction in insulin levels leads to glucose overload, either due to increased consumption of glucose or by an increased level of counter-regulatory hormones which include catecholamines, cortisol, glucagon, and growth hormone. This imbalance results in increased glucose production by the liver with resistance in glucose utilization in the peripheral tissues [3]. Diabetic ketoacidosis also impairs metabolic processes causing increased gluconeogenesis, lipolysis, ketogenesis, and decreased glycolysis [5].

In our study, diabetic ketoacidosis was more prevalent in type 2 diabetes. The most common clinical presentation was nausea and vomiting, followed by abdominal pain. Seth et al. in 2015 also reported nausea, vomiting, and pain in abdomen as the most common clinical presentations in patients with DKA [3]. Severe vomiting and abdominal pain are reported as the most common symptoms in other studies as well $[2,6]$. Ongoing catabolism and acidosis in DKA patients can lead to extreme vomiting [7]. In this study, dehydration was present in $30 \%$ of the participants. Osmotic diuresis caused by glycosuria is mainly responsible for dehydration and electrolyte disturbance [7].

In our study, infection and non-compliance to treatment were major precipitating factors. This result is similar to other studies, which also reported infections and non-compliance as major precipitating factors $[2,3]$. These precipitating factors are particularly important as both infection and non-compliance are common in patients with diabetes. The greater incidence of infection in diabetic patients is due to numerous factors including damage to neutrophil function, impairment of humoral immune system, and neuropathies [8]. In developed countries, the rate of non-compliance in long-term diabetic patients is $50 \%$. World Health Organization (WHO) predicts that the rate of non-compliance to diabetic treatment may be even higher in developing countries [9]. According to a study in Pakistan, $62 \%$ of the diabetic population is non-compliant to their treatment regime [10]. Various studies have identified other factors as well which are responsible for diabetic ketoacidosis. These factors include events such as myocardial infarction, pulmonary embolism, and pancreatitis as well as the use of alcohol and drugs [7].

In this study, pneumonia (38.7\%) and urinary tract infection (30.6\%) were the most common infections identified in patients with DKA. Seth et al. also reported that pneumonia and urinary tract infection were common in diabetic ketoacidosis patients [3]. Apart from these infections, patients also presented with 
tuberculosis, diabetic foot, and gastrointestinal tract infections. This was in accordance with previous studies, which showed that infection in any part of the body may result in diabetic ketoacidosis [11,12].

To the best of our knowledge, this is the first study from a rural area of Pakistan that has studied clinical presentation and precipitating factors of DKA. It will help clinicians identify high-risk patients for diabetic ketoacidosis and give them time to prepare for medical intervention and if needed, to prevent mortality. However, we are aware of the limitations of the study. Since it was carried out in an emergency department, after initial management patients were shifted to either ward or ICU and were not followed for outcome. The study was only conducted in one institute, hence we cannot generalise the results.

Diabetic ketoacidosis is not an infrequent complication of diabetic mellitus. The clinical presentation is vague. Symptoms that should raise suspicion regarding DKA include nausea, vomiting, abdominal pain, and dehydration. Appropriate diagnostic tests should be done for timely diagnosis of diabetic ketoacidosis.

\section{Conclusions}

In our study, diabetic ketoacidosis was more prevalent in type 2 diabetes. Patients commonly presented with nausea, vomiting, and abdominal pain. The most common precipitating factors were infections (such as pneumonia and urinary tract infections) and non-compliance to treatment. Awareness among diabetic patients and physicians regarding symptoms and precipitating factors of diabetic ketoacidosis is necessary, as it may assist in early diagnosis and timely treatment.

\section{Additional Information \\ Disclosures}

Human subjects: Consent was obtained by all participants in this study. Ghulam Muhammad Mahar Medical College issued approval GMMMC/0619/3B. Animal subjects: All authors have confirmed that this study did not involve animal subjects or tissue. Conflicts of interest: In compliance with the ICMJE uniform disclosure form, all authors declare the following: Payment/services info: All authors have declared that no financial support was received from any organization for the submitted work. Financial relationships: All authors have declared that they have no financial relationships at present or within the previous three years with any organizations that might have an interest in the submitted work. Other relationships: All authors have declared that there are no other relationships or activities that could appear to have influenced the submitted work.

\section{References}

1. Umpierrez G, Korytkowski M: Diabetic emergencies-ketoacidosis, hyperglycaemic hyperosmolar state and hypoglycaemia. Nat Rev Endocrinol. 2016, 12:222-32. 10.1038/nrendo.2016.15

2. Ahuja W, Kumar N, Kumar S, et al.: Precipitating risk factors, clinical presentation, and outcome of diabetic ketoacidosis in patients with type 1 diabetes. Cureus. 2019,11:4789. 10.7759/cureus.4789

3. Seth P, Kaur H, Kaur M: Clinical profile of diabetic ketoacidosis: a prospective study in a tertiary care hospital. J Clin Diagn Res. 2015, 9:01-4. 10.7860/JCDR/2015/8586.5995

4. Barski L, Nevzorov R, Rabaev E, et al.: Diabetic ketoacidosis: clinical characteristics, precipitating factors and outcomes of care. Isra Med Assoc J. 2012, 14:299-303.

5. Kitabchi AE, Umpierrez GE, Murphy MB, Kreisberg RA: Hyperglycemic crises in adult patients with diabetes: a consensus statement from the American Diabetes Association. Diabetes Care. 2006, 29:2739-48. 10.2337/dc06-9916

6. Shaltout AA, Channanath AM, Thanaraj TA, et al.: Ketoacidosis at first presentation of type 1 diabetes mellitus among children: a study from Kuwait. Sci Rep. 2016, 6:27519. 10.1038/srep27519

7. Gosmanov AR, Gosmanova EO, Kitabchi AE: Hyperglycemic crises: diabetic ketoacidosis (DKA), and hyperglycemic hyperosmolar state (HHS). Endotext. Feingold KR, Anawalt B, Boyce A, et al. (ed): MDText.com, Inc., South Dartmouth; 2018.

8. Casqueiro J, Casqueiro J, Alves C: Infections in patients with diabetes mellitus: a review of pathogenesis . Indian I Endocrinol Metab. 2012, 16:27-36. 10.4103/2230-8210.94253

9. In Chronic Diseases - Poor compliance of Patients with drug treatment . http://www.biomedicine.org/medicine-news/In-Chronic-Diseases---Poor-compliance-of-Patients-with-drug-treatment-2097-1/.

10. Shams N, Amjad S, Kumar N, Ahmed W, Saleem F: Drug non-adherence in type 2 diabetes mellitus; predictors and associations. J Ayub Med Coll Abbottabad. 2016, 28:302-307.

11. Westphal SA: The occurrence of diabetic ketoacidosis in non-insulin dependent diabetes and newly diagnosed diabetic adults. Am J Med. 1996, 101:19-24. 10.1016/s0002-9343(96)00076-9

12. Umpierrez GE, Khajavi M, Kitabchi AE: Review: diabetic ketoacidosis and hyperglycaemic hyperosmolar nonketotic syndrome. Am J Med Sci. 1996, 311:225-33. 10.1097/00000441-199605000-00006 